\title{
A PRELIMINARY STUDY ON THE BIRDS OF THANE CREEK, MAHARASHTRA, INDIA
}

\author{
Sheetal Chaudhari-Pachpande ${ }^{1}$ \& Madhuri K. Pejaver ${ }^{2}$ \\ 1,2 Department of Zoology,VPM's B N Bandodkar College of Science affiliated with the University of Mumbai, \\ 'Inanadweepa', College Campus, Chendani Bunder Road, Thane (West), Maharashtra 400601, India \\ ${ }^{1}$ sheetalpachpande@gmail.com (corresponding author), ${ }^{2}$ mkpejaver@gmail.com
}

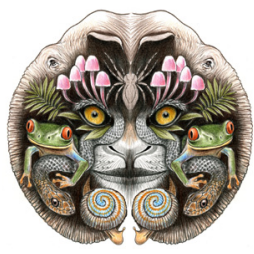

ISSN 0974-7907 (Online) ISSN 0974-7893 (Print)

\section{OPEN ACCESS}

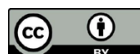

Abstract: Shorebirds also known as waders comprise severa adaptations, which enable them to forage on exposed mudflats. The population of birds in any ecosystem shows the environmental quality of the area, pollution level, security and availability of food and habitat. Thane Creek located in Mumbai is one of the unique mangrove ecosystems, maintaining a good population of sediment-dwelling organisms that support a myriad of migratory and non-migratory bird populations. Bird surveys were carried out using the point count method across two different locations at Thane Creek. In total 95 species of birds were recorded during the study and distinguished as per the pattern of their foraging. A healthy diversity of bird species observed indicates the high productivity of the creek.

Keywords: Avifauna, Thane Creek, mudflats, productivity, mangroves, ecosystem

India hosts rich coastal and marine biodiversity in various wetland habitats such as creeks, mangroves, mudflats and salt marshes. Mangroves provide an important habitat for a variety of planktonic and benthic organisms (Nagelkerkan 2008) which attract a myriad of migratory and non-migratory birds that utilize the mangrove ecosystem in varying degrees from feeding, roosting, to breeding (Oswin 2002). The mudflats of the mangrove ecosystems are reported to play a significant role in the conservation of resident birds, migratory and endangered birds (Pawar 2011). Most of the birds that visit mudflats possess long legs and toes, long and sometimes curved bills and are called waders. These adaptations enable the waders to feed in shallow water habitats.

Waders are an important component of the mangrove ecosystem and their occurrence and distribution help to understand the overall picture of the wetland habitat. Most of the birds have specific habitat requirements from season to season, a loss of which may lead to their extinction (Chauhan et al. 2008). Ali \& Ripley (1987) reported that India harbors 1,340 bird species which decreased over a decade to 1,237 species (Daniels 1997). Habitat destruction is considered as one of the main reason for such a decrease in bird species population. Presently wetlands are reported as highly fragile ecosystem due to increasing anthropogenic stress. Therefore, this calls for an urgent need for conservation of bird habitats to sustain their population. Wetlands are known to support over 310 species (Kumar et al. (2005). Thane Creek located between Mumbai and Navi Mumbai, inhabits a large diversity of avifauna and is regarded as one of the Important Bird Areas (Islam \& Rahmani 2004), as over 205 species of birds are reported from Thane Creek alone (Nitsure 2002).

DOI: http://dx.doi.org/10.11609/jott.2397.8.5.8797-8803 | ZooBank: urn:Isid:Zoobank.org:pub:E2B4BF84-9C44-40D0-87A3-DA45B51094FA

Editor: S. Balachandran, Bombay Natural History Society, Mumbai, India.

Date of publication: 26 May 2016 (online \& print)

Manuscript details: Ms \# 2397 | Received 12 March 2015 | Final received 04 July 2016 | Finally accepted 10 May 2016

Citation: Chaudhari-Pachpande, S. \& M.K. Pejaver (2016). A preliminary study on the birds of Thane Creek, Maharashtra, India. Journal of Threatened Taxa 8(5): 8797-8803; http://dx.doi.org/10.11609/jott.2397.8.5.8797-8803

Copyright: (c) Chaudhari-Pachpande \& Pejaver 2016. Creative Commons Attribution 4.0 International License. JoTT allows unrestricted use of this article in any medium, reproduction and distribution by providing adequate credit to the authors and the source of publication.

Funding: None.

Conflict of Interest: The authors declare no competing interests.

Acknowledgments: The authors wish to thank the management of B. N. Bandodkar College of Science and Mr.Yashwant Pawar and Namdeo Pawar, local fishermen, for their support and encouragement during research work. 
Bilgrami (1995) stated avifaunal diversity as an essential ecological tool to evaluate the health of different habitats both qualitatively and quantitatively. Wiens (1995) stated that due to the fragmentation of habitats, the viability of the wildlife population is at threat and the same is true for waterbirds and their habitats, the wetlands. According to Kumar et al. (2003, 2005) baseline information is a prerequisite for planning and monitoring management actions for aquatic birds as well as their habitats. Several researchers believed that monitoring of birds of a locality is crucial in predicting the ecological health and productivity of wetlands (Furness \& Greenwood 1993; Newton 1995; Ali 1996; Chamberlain et al. 2007; Li et al. 2009; Rotenberry \& Wiens 2009). Thane Creek being an important Bird Area, it is necessary to monitor the diversity of birds to predict the health of the ecosystem. The data related to bird diversity at Thane Creek is scarce. Therefore, the present paper depicts the diversity of different species of waders recorded from two stations located at Thane Creek.

\section{MATERIALS AND METHODS}

\section{Study Area}

Thane Creek is a $26 \mathrm{~km}$ long inlet from the Arabian Sea towards the landward side near the city of Thane, Mumbai and Navi Mumbai with luxuriant mangrove vegetation and exposed mudflats along its bank (Fig. 1). The area has a moderate humid tropical climate with a maximum temperature of $40^{\circ} \mathrm{C}$ and a minimum temperature of $16^{\circ} \mathrm{C}$. In spite of several anthropogenic stresses, the mudflats of the Creek fulfil the food requirement for diversity of bird species mainly waders. The mudflats exposed during low tide are used as the foraging grounds by a variety of wading birds mainly a huge flock of lesser flamingo, greater flamingos and a multitude of water birds during the winter season.

One station was selected on each bank of Thane Creek located in close proximity to Bhandup and Airoli villages respectively. Station I selected at Bhandup is located adjacent to the Bhandup Pumping Station which receives waste water from the Mumbai metropolitan area. It also harbors a few abandoned aquaculture ponds built by the local fishing community and serves as the best location for spotting birds for bird watchers. The area supports a rich avifaunal diversity as it provides a mix of different vegetation in small patches that include grasslands, water bodies, mangrove, and mudflats. Station II is situated near Airoli Village with active aquaculture ponds, wetland vegetation namely Avicennia marina, Excoecaria agallocha, Sonneratia apetala. The data was collected for a period of 18 months from September 2009 to April 2011 during spring tides and neap tides. The vegetation of the study area includes dominant mangrove species Avicennia marina var. accutissima Stapf ex Moldenke and other less dominant species such as Avicennia officinalis Linn., Sonneratia apetala BuchHam, Sonneratia alba J. Smith, Bruguiera cylindrica (L.) Blume, Aegeiceras corniculatum (L.) Blanco, Excoecaria agallocha Linn.and associate mangrove species such as Acanthus iillicifolius Linn., Salvadora persica (L.) Garcin, Derris trifoliata Lour., Sesuvium portulacastrum Linn., and patches of mangrove grass namely Aleuropus lagopoides (Linn.) Trin. Ex Thw. The data obtained from
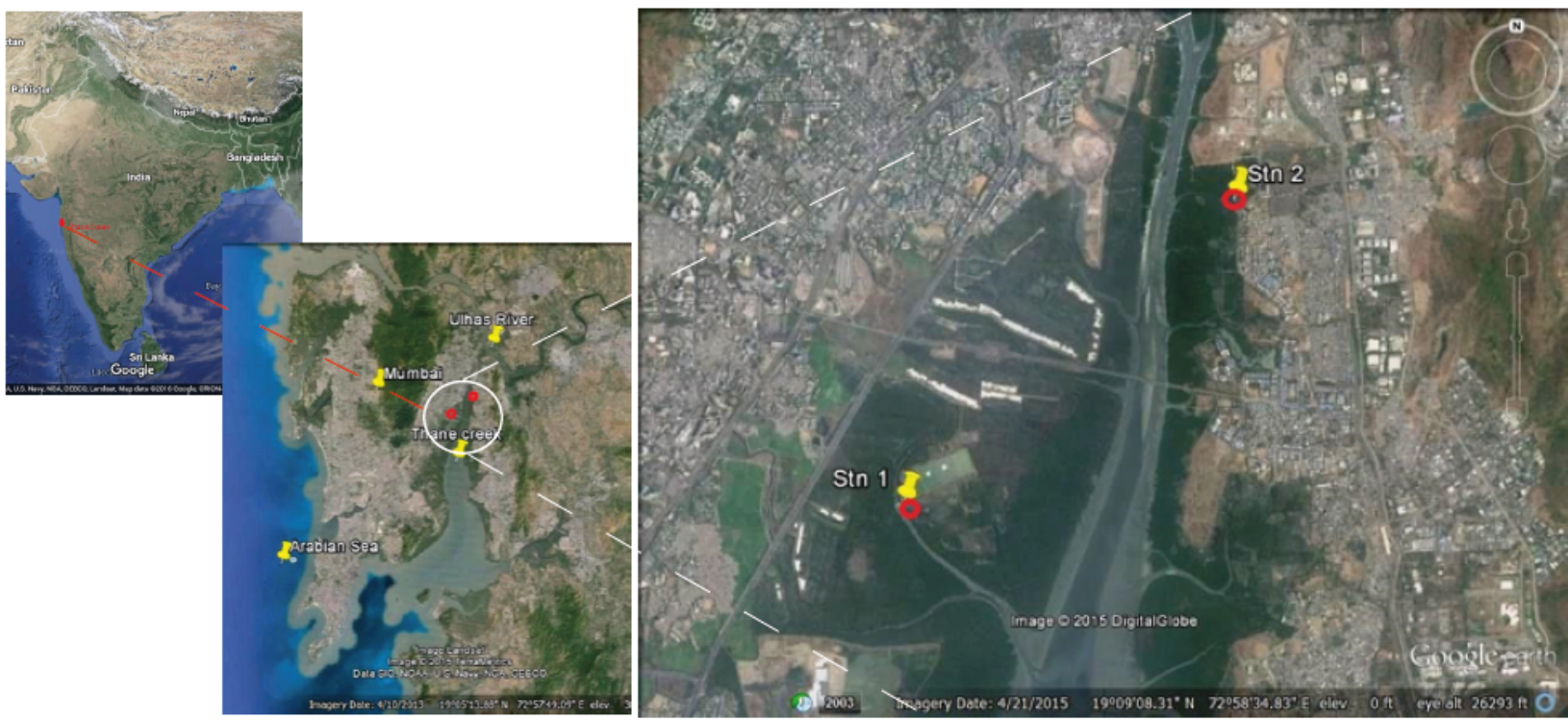

Figure 1. Location of study area across Thane Creek. 
the two stations is pooled and displayed in Table 1.

\section{MethodS}

Birds are counted following a wide variety of methods (Bibby 2000). For the present study, point counting was done using binoculars (Olympus $10 \times 50$ ). In this method, a suitable vantage point was selected, and all visible birds were counted. Another method, "total count" was used wherever possible, by walking around the wetlands from the landward side or to specific vantage points to count the birds (Vijayan 1991). A total area of $6 \mathrm{~km}$ was investigated for the study.

Bird species observed in the field were identified using standard field guides (Kazmierczak 2000; Grimmet 2013). Photographs were obtained using Canon SX5 whenever and wherever possible. The data was bird species which were grouped under various categories namely, frugivorous, insectivorous, carnivorous, omnivorous depending on their feeding preferences.

\section{OBSERVATIONS}

In total 95 species of birds were recorded during the study period. All the birds recorded at the selected stations were further categorized according to their feeding behavior to study the preferences of foraging habitats selected by various birds visiting the creek. The bird species were further classified as resident, migratory, and resident migratory bird species (Table 1).

The vegetation of the mangrove ecosystem at Thane Creek supported the population of both waders and terrestrial bird species. A maximum diversity of bird species was reported at Bhandup (Station I) as it provides a mixed habitat that includes Saltpans, grasslands, planted trees, and mangrove vegetation. The strong plant-animal (birds) interaction was observed for Whitecheeked Bulbul which were seen in plenty on Salvadora persica during the fruiting season and Baya Weavers were observed in large numbers nesting on Sonneratia apetala.

Species such as Indian Skimmer (Vulnerable and rare), Red-headed Bunting (rare), Eurasian Collared Dove (rare), White Stork (rare) were reported at station I. The near threatened species namely Painted Stork, Lesser Flamingo, Black-headed Ibis (IUCN 2015) were observed during the migratory season from December to March at both the stations.

The distribution of birds as per their feeding behaviour revealed a maximum population of carnivorous (49\%), followed by omnivorous (26\%), insectivorous (18\%), Granivorous (4\%), Nectarivorous (2\%) and Frugivorous (1\%) (Fig. 2). Habitat-wise distribution revealed that

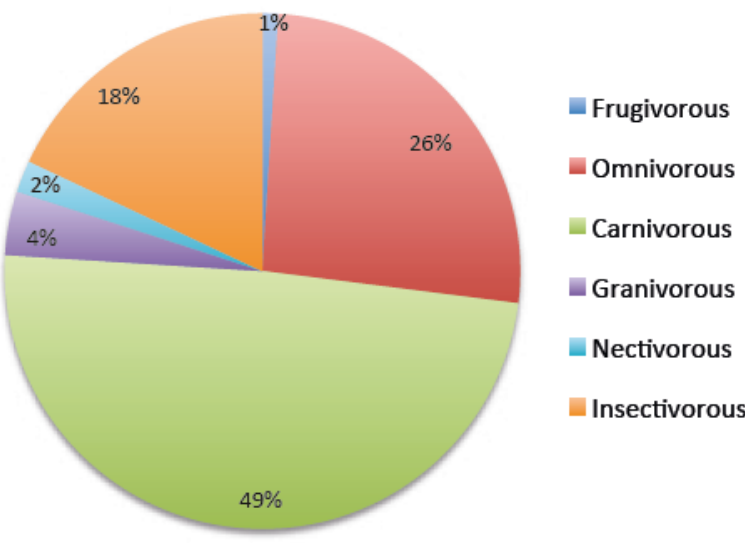

Figure 2. Distribution of birds according to their feeding habit

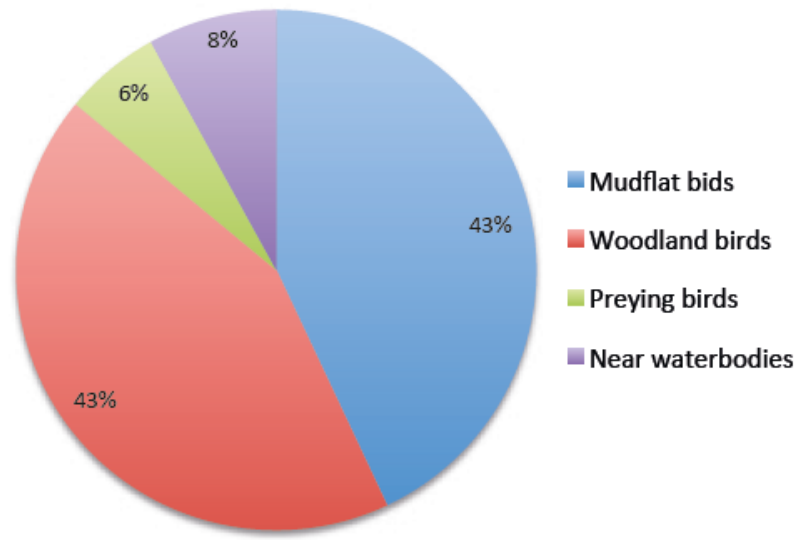

Figure 3. Distribution of birds according to habitat use for feeding

$43 \%$ of birds were dependent on mudflats and $43 \%$ birds on woodland made up of mainly mangroves. This suggested that $86 \%$ of birds are dependent on mangroves and mudflats (Fig. 3).

The data revealed that the small patch of approximately $6 \mathrm{~km}$ of mangrove ecosystem of Thane Creek harbors a fairly large number of bird population and any disturbance of such habitat might affect the avifaunal communities to a large extent. The creek is under severe threat today due to increased anthropogenic usage of wetlands, water pollution, drainage of wetlands, an agricultural conversion that leads to disturbance to nesting sites and habitat destruction.

\section{CONCLUSION}

The vegetation structure of a mangrove ecosystem and mudflats of Thane Creek are used by waders as shelter belt, foraging, roosting and other purposes during spring migration. Most of the birds have specific habitat requirements from season to season, a loss of which may lead to their local extinction. Past 
Table 1. The detailed combined list of birds observed at Station I and Station II in Thane Creek

\begin{tabular}{|c|c|c|c|c|c|c|c|}
\hline & Common name & Scientific name & Habitat & $\begin{array}{l}\text { Population } \\
\text { Status }\end{array}$ & $\begin{array}{l}\text { IUCN } \\
\text { status }\end{array}$ & $\begin{array}{l}\text { C, } \\
U C, R\end{array}$ & $\begin{array}{l}\text { S. S } \\
* * *\end{array}$ \\
\hline & \multicolumn{7}{|l|}{ Anatidae } \\
\hline \multirow[t]{2}{*}{1} & Indian Spot-billed Duck & Anas poecilorhyncha & Mangroves, marshes & ++ & LC & C & RM \\
\hline & \multicolumn{7}{|l|}{ Ciconiidae } \\
\hline 2 & Painted Stork & Mycteria leucocephala & Aquatic grass, marshes & ++ & NT & C & RM \\
\hline \multirow[t]{2}{*}{3} & White Stork & Ciconia ciconia & Aquatic grass, marshes & + & LC & $U C, R$ & M \\
\hline & \multicolumn{7}{|l|}{ Phoenicopteridae } \\
\hline 4 & Greater Flamingo & Phoenicopterus roseus & Creeks, saltpans & ++ & LC & C & RM \\
\hline \multirow[t]{2}{*}{5} & Lesser Flamingo & Phoenicopterus minor & Creeks, saltpans & +++ & NT & C & RM \\
\hline & Threskiornithidae & & & & & & \\
\hline 6 & Black-headed Ibis & Threskiornis melanocephalus & Aquatic grass, marshes & ++ & NT & C & RM \\
\hline 7 & Glossy Ibis & Plegadis falcinellus & Aquatic grass, marshes & ++ & LC & $\mathrm{C}$ & RM \\
\hline \multirow[t]{2}{*}{8} & Eurasian Spoonbill & Platalea leucorodia & Aquatic grass, creeks, mangroves & ++ & LC & C & RM \\
\hline & Ardeidae & & & & & & \\
\hline 9 & Black-crowned Night Heron & Nycticorax nycticorax & $\begin{array}{l}\text { Mangroves saltpan, marsh, } \\
\text { aquatic grass }\end{array}$ & + & LC & UC & $\mathrm{R}$ \\
\hline 10 & Indian Pond Heron & Ardeola grayii & $\begin{array}{l}\text { Mangroves saltpan, marsh, } \\
\text { aquatic grass }\end{array}$ & +++ & LC & C & $\mathrm{R}$ \\
\hline 11 & Straited Heron & Butorides striata & Manroves, marsh & ++ & LC & UC & $\mathrm{R}$ \\
\hline 12 & Grey Heron & Ardea cinerea & Creeks, marshes & ++ & LC & C & RM \\
\hline 13 & Purple Heron & Ardea purpurea & Aquatic grass & + & LC & UC & $\mathrm{R}$ \\
\hline 14 & Cattle Egret & Bubulcus ibis & Crops, aquatic grass, marsh & +++ & LC & C & RM \\
\hline 15 & Great Egret & Casmerodius albus & Crops, aquatic grass, marsh & ++ & LC & C & $\mathrm{R}$ \\
\hline 16 & Intermediate Egret & Mesophoyx intermedia & Crops, aquatic grass, marsh & +++ & NK & C & $\mathrm{R}$ \\
\hline 17 & Little Egret & Egretta garzetta & Crops,mangroves & +++ & LC & C & $\mathrm{R}$ \\
\hline \multirow[t]{2}{*}{18} & Western Reef Egret & Egretta gularis & Creeks & ++ & LC & C & RM \\
\hline & Phalacrocorcidae & & & & & & \\
\hline 19 & Little Cormorant & Phalacrocorax niger & Open water bodies & +++ & LC & C & RM \\
\hline \multirow[t]{2}{*}{20} & Great Cormorant & Phalacrocorax carbo & Open water bodies & ++ & LC & C & M \\
\hline & Accipitridae & & & & & & \\
\hline 21 & Black Kite & Milvus migrans & Mangroves, marshes, saltpans & +++ & LC & C & $\mathrm{R}$ \\
\hline 22 & Brahminy Kite & Haliastur indus & Mangroves, marshes, saltpans & ++ & LC & $\mathrm{C}$ & $\mathrm{R}$ \\
\hline 23 & Black-winged Kite & Elanus caeruleus & Mangroves & + & LC & UC & $\mathrm{R}$ \\
\hline 24 & Osprey & Pandion haliaetus & Creeks & + & LC & UC & RM \\
\hline 25 & Eurasian Marsh Harrier & Circus aeruginosus & $\begin{array}{l}\text { Mangroves, aquatic grasses, } \\
\text { marshes, saltpans }\end{array}$ & ++ & LC & $\mathrm{C}$ & M \\
\hline \multirow[t]{2}{*}{26} & Shikra & Accipiter badius & Woodland, mangroves & + & LC & UC & $\mathrm{R}$ \\
\hline & Rallidae & & & & & & \\
\hline \multirow[t]{2}{*}{27} & White-breasted Waterhen & Amaurornis phoenicurus & Mangroves & ++ & LC & C & $\mathrm{R}$ \\
\hline & Charadriidae & & & & & & \\
\hline 28 & Little Ringed Plover & Charadrius dubius & Marsh, saltpans, creeks & ++ & LC & C & RM \\
\hline 29 & Kentish Plover & Charadrius alexandrinus & Marsh, saltpans, creeks & ++ & LC & C & RM \\
\hline 30 & Lesser Sand Plover & Charadrius mongolus & Marsh, saltpans, creeks & ++ & LC & UC & RM \\
\hline \multirow[t]{2}{*}{31} & Red-wattled Lapwing & Vanellus indicus & Marsh,mangroves, saltpans & + & LC & C & $\mathrm{R}$ \\
\hline & Recurvirostridae & & & & & & \\
\hline 32 & Black-winged Stilt & Himanto pushimantopus & Marsh, saltpans, creeks & +++ & LC & C & $\mathrm{R}$ \\
\hline 33 & Pied Avocet & Recurviro straavosetta & Open waterbody & +++ & LC & C & RM \\
\hline
\end{tabular}




\begin{tabular}{|c|c|c|c|c|c|c|c|}
\hline & Common name & Scientific name & Habitat & $\begin{array}{l}\text { Population } \\
\text { Status }\end{array}$ & $\begin{array}{l}\text { IUCN } \\
\text { status }\end{array}$ & $\begin{array}{c}C, \\
U C, R\end{array}$ & $\begin{array}{l}\text { S. S } \\
* * *\end{array}$ \\
\hline & Scolopacidae & & & & & & \\
\hline 34 & Black-tailed Godwit & Limosa limosa & Marsh, saltpans, creeks & ++ & NT & C & M \\
\hline 35 & Eurasian Curlew & Numenius arquata & Marsh, saltpans, creeks & ++ & NT & C & M \\
\hline 36 & Common Redshank & Tringa totanus & Marsh, saltpans, creeks & +++ & LC & C & M \\
\hline 37 & Marsh Sandpiper & Tringa stagnatilis & Marsh, saltpans, creeks & ++ & LC & C & M \\
\hline 38 & Wood Sand piper & Tringa glareola & Marsh, saltpans, creeks & ++ & LC & C & M \\
\hline 39 & Common Sandpiper & Actitis hypoleucos & $\begin{array}{l}\text { Marsh, saltpans, creeks, aquatic } \\
\text { grasses }\end{array}$ & ++ & LC & C & M \\
\hline \multirow[t]{2}{*}{40} & Little Stint & Calidris minuta & Marsh, saltpans, creeks & ++ & LC & C & M \\
\hline & Laridae & & & & & & \\
\hline 41 & Brown-headed Gull & Chroicocephalus brunnicephalus & Marsh, saltpans, creeks & +++ & LC & C & M \\
\hline 42 & Black-headed Gull & Chroicocephalus ridibundus & Marsh, saltpans, creeks & ++ & LC & C & M \\
\hline 43 & Caspian Tern & Hydroprogne caspia & Creek & +++ & LC & C & M \\
\hline 44 & River Tern & Sterna aurantia & Creek & + & NT & UC & RM \\
\hline 45 & Whiskered Tern & Chlidonias hybrida & Marsh, saltpan & ++ & LC & c & RM \\
\hline \multirow[t]{2}{*}{46} & Indian Skimmer & Rynchops albicollis & Marsh, saltpan & + & v & UC & M \\
\hline & Columbidae & & & & & & \\
\hline 47 & Common Pigeon & Columba livia & Woodland & + & NK & C & $\mathrm{R}$ \\
\hline 48 & Spotted Dove & Stigmatopelia chinensis & Mangroves, woodland & ++ & NK & C & $\mathrm{R}$ \\
\hline \multirow[t]{2}{*}{49} & Eurasian Collared Dove & Streptopelia orientalis & Woodland, mangroves & + & LC & UC,R & $\mathrm{R}$ \\
\hline & Cuculidae & & & & & & \\
\hline 50 & Asian Koel & Eudynamys scolopaceus & Mangroves, woodland & + & LC & c & $\mathrm{R}$ \\
\hline \multirow[t]{2}{*}{51} & Southern Coucal & Centropus (sinensis) parroti & Mangroves & ++ & LC & C & $\mathrm{R}$ \\
\hline & Apodidae & & & & & & \\
\hline \multirow[t]{2}{*}{52} & Alpine Swift & Tachymarptis melba & Mangroves & ++ & LC & c & $\mathrm{R}$ \\
\hline & Alcedinidae & & & & & & \\
\hline 53 & White-throated Kingfisher & Halcyon smyrnensis & Along water bodies & ++ & LC & c & $\mathrm{R}$ \\
\hline 54 & Black-capped Kingfisher & Halcyon pileata & Along water bodies & + & LC & $U C, R$ & M \\
\hline 55 & Common Kingfisher & Alcedo atthis & Along water bodies & ++ & NK & C & $\mathrm{R}$ \\
\hline \multirow[t]{2}{*}{56} & Pied Kingfisher & Ceryle rudis & Along water bodies & + & LC & c & $\mathrm{R}$ \\
\hline & Meropidae & & & & & & \\
\hline 57 & Green Bee-eater & Merops orientalis & Mangroves, grass & ++ & LC & c & M \\
\hline \multirow[t]{2}{*}{58} & Blue-tailed Bee-eater & Merops philippinus & Mangroves grass & +++ & LC & C & M \\
\hline & Ramphastidae & & & & & & \\
\hline \multirow[t]{2}{*}{59} & Coppersmith Barbet & Megalaima haemacephalus & Plantation & + & LC & C & $\mathrm{R}$ \\
\hline & Picidae & & & & & & \\
\hline \multirow[t]{2}{*}{60} & Eurasian Wryneck & Jynx torquilla & Mangroves & + & LC & UC & M \\
\hline & Aegithinidae & & & & & & \\
\hline \multirow[t]{2}{*}{61} & Common lora & Aegithina tiphia & Mangroves & + & LC & c & $\mathrm{R}$ \\
\hline & Laniidae & & & & & & \\
\hline \multirow[t]{2}{*}{62} & Long-tailed Shrike & Lanius schach & Mangroves & ++ & LC & C & $\mathrm{R}$ \\
\hline & Dicruridae & & & & & & \\
\hline \multirow[t]{2}{*}{63} & Black Drongo & Dicrurus macrocercus & Grass, woodland & + & LC & C & $\mathrm{R}$ \\
\hline & Oriolidae & & & & & & \\
\hline \multirow[t]{2}{*}{64} & Indian Golden Oriole & Oriolus(oriolus) kundoo & Woodland & + & LC & C & RM \\
\hline & Rhipiduridae & & & & & & \\
\hline 65 & White-browed Fantail & Rhipidura aureola & Mangroves & + & LC & C & $\mathrm{R}$ \\
\hline
\end{tabular}




\begin{tabular}{|c|c|c|c|c|c|c|c|}
\hline & Common name & Scientific name & Habitat & $\begin{array}{l}\text { Population } \\
\text { Status }\end{array}$ & $\begin{array}{l}\text { IUCN } \\
\text { status }\end{array}$ & $\begin{array}{c}\text { C, } \\
\text { UC,R }\end{array}$ & $\begin{array}{l}\text { S. S } \\
* * *\end{array}$ \\
\hline & Corvidae & & & & & & \\
\hline 66 & Indian Jungle Crow & $\begin{array}{l}\text { Corvus(macrorhynchos) } \\
\text { culminatus }\end{array}$ & Mangroves, woodland & +++ & LC & C & $\mathrm{R}$ \\
\hline \multirow[t]{2}{*}{67} & House Crow & Corvus splendens & Mangroves, wooland, grass & +++ & LC & C & $\mathrm{R}$ \\
\hline & Hirundinidae & & & & & & \\
\hline 68 & Wire-tailed Swallow & Hirundo smithii & Saltpans, along nullah, mangroves & ++ & LC & C & LM \\
\hline \multirow[t]{2}{*}{69} & Barn Swallow & Hirundo rustica & Saltpans, along nullah, mangroves & ++ & LC & C & RM \\
\hline & Pycnonotidae & & & & & & \\
\hline 70 & Red-whiskered Bulbul & Pycnonotus jocosus & Mangroves & ++ & LC & c & $\mathrm{R}$ \\
\hline 71 & White-eared Bulbul & Pycnonotus leucotis & Mangroves & ++++ & LC & C & $\mathrm{R}$ \\
\hline \multirow[t]{2}{*}{72} & Red-vented Bulbul & Pycnonotus cafer & Mangroves & + & LC & UC & $\mathrm{R}$ \\
\hline & Cisticolidae & & & & & & \\
\hline 73 & Ashy Prinia & Prinia socialis & Mangroves & ++ & LC & C & $\mathrm{R}$ \\
\hline 74 & Plain Prinia & Prinia inornata & Mangroves & ++ & LC & C & $\mathrm{R}$ \\
\hline \multirow[t]{2}{*}{75} & Common Tailorbird & Orthoto mussutorius & Mangroves & ++ & LC & C & $\mathrm{R}$ \\
\hline & Sylviidae & & & & & & \\
\hline 76 & Clamorous Reed Warbler & Acrocephalus stentoreus & Mangroves & + & LC & UC & $\mathrm{R}$ \\
\hline \multirow[t]{2}{*}{77} & Common Chiffchaff & Phylloscopus collybita & Woodland, mangroves & ++ & LC & UC & $M$ \\
\hline & Zosteropidae & & & & & & \\
\hline \multirow[t]{2}{*}{78} & Oriental White-eye & Zosterops palpebrosus & Mangroves & ++ & LC & UC & $\mathrm{R}$ \\
\hline & Sturnidae & & & & & & \\
\hline 79 & Rosy Starling & Pastor roseus & Mangroves, woodland & ++ & LC & C & M \\
\hline 80 & Asian Pied Starling & Gracupica contra & Grass woodland & ++ & LC & C & $\mathrm{R}$ \\
\hline 81 & Common Myna & Acridotheres tristis & Grass, woodland, mangroves & ++ & LC & C & $\mathrm{R}$ \\
\hline \multirow[t]{2}{*}{82} & Brahminy Starling & Sturnia pagodarum & Woodland & ++ & LC & C & $R$ \\
\hline & Muscicapidae & & & & & & \\
\hline 83 & Bluethroat & Luscinia svecica & Woodland, mangroves & + & LC & UC & $R$ \\
\hline 84 & Oriental Magpie-Robin & Copsychus saularis & Woodland, mangroves & ++ & LC & C & $R$ \\
\hline 85 & Indian Robin & Saxicoloides fulicatus & Woodland, mangroves & ++ & LC & C & $\mathrm{R}$ \\
\hline 86 & Desert Wheatear & Oenanthe deserti & Woodland, mangroves & + & LC & UC & M \\
\hline \multirow[t]{2}{*}{87} & Common Stonechat & Saxicolator quatus & Woodland, mangroves & + & NK & UC & M \\
\hline & Nectariniidae & & & & & & \\
\hline 88 & Purple Sunbird & Cinnyris asiaticus & Woodland, mangroves & + & LC & c & $\mathrm{R}$ \\
\hline \multirow[t]{2}{*}{89} & Purple-rumped Sunbird & Leptocoma zeylonica & Woodland, mangroves & ++ & LC & C & $\mathrm{R}$ \\
\hline & Passeridae & & & & & & \\
\hline \multirow[t]{2}{*}{90} & House Sparrow & Passer domesticus & Woodland, mangroves & ++ & LC & C & R \\
\hline & Ploceidae & & & & & & \\
\hline \multirow[t]{2}{*}{91} & Baya Weaver & Ploceus philippinus & Mangroves & +++ & LC & C & R \\
\hline & Estrildidae & & & & & & \\
\hline 92 & Red Avadavat & Amandava amandava & Mangroves, woodland & +++ & LC & c & $\mathrm{R}$ \\
\hline \multirow[t]{2}{*}{93} & Scaly-breasted Munia & Lonchura punctulata & Woodland & +++ & LC & C & $\mathrm{R}$ \\
\hline & Motacillidae & & & & & & \\
\hline \multirow[t]{2}{*}{94} & Yellow Wagtail & Motacilla flava & Aquatic grasses, near water bodies & ++ & LC & C & RM \\
\hline & Emberizidae & & & & & & \\
\hline 95 & Red-headed Bunting & Emberiza bruniceps & Woodland & + & LC & $U C, R$ & M \\
\hline
\end{tabular}

IUCN status: LC - Least Concerned; NT - Near Threatened. Seasonal status (s.s) *** m - migrant, $r$ - resident, rm - resident migrant

Population status: ++++ abundant, +++ medium, ++ average, + meager, - absent. $C=$ common, $u c=$ uncommon, $r=$ rare 
and present researchers stated that, Thane Creek is heavily polluted with wastes from both industrial and domestic sources discharged into the creek through several outlets. Regardless of which, it still supports thousands of birds of 95 species, including the small waders and the flamingos. Flamingoes serve to be the major attraction of the creek that has led the forest department to declare certain parts of the creek as Flamingo Sanctuary. The present study area includes this part of the sanctuary and needs especial attention for improving and conserving the condition of the creek. Building of watchtowers, generating awareness about birds and their role in maintaining the balance of the ecosystem needs to be highlighted among the local masses which might be helpful to save the creek from severe anthropogenic stress.

\section{References}

Arunkumar, J.P. Sati \& P.C. Tak (2003). Check list of Indian Waterbirds; Envis News letter: Avian Ecology \& Inland wetlands. Buceros 8(1): $1-29$.

Ali, S. \& S.D. Ripley (1987). Compact Handbook of the Birds of India and Pakistan Together with those of Bangladesh, Nepal, Bhutan and Sri Lanka. Oxford University Press, Delhi, 890pp.

Ali, S. (1996). The Book of Indian Birds. Oxford university press, New Delhi, 466pp.

Bibby C.J., N.D. Burgess \& D.A. Hill (1992). Bird Census Techniques. Academic Press, London, 67-84 pp.

Bilgrami K.S. (1995). Concept and Conservation of Biodiversity. CBS Publishers and Distributors, Delhi.

Chauhan, R.R., H.U. Shingadia \& V. Sakthivel (2008). Survey of avifauna of Borivali mangroves along the coast of Mumbai. Nature Environmental and Pollution Technology 7(2): 229-233.

Chamberlain, D.E., M.P. Toms, M.R. Cleary \& A.N. Banks (2007). House Sparrow (Passer domesticus) habitat use in urbanized landscapes. Journal of Ornithology 148: 453-462.

Daniels, R.J.R. (1997). A Field Guide to the Birds of South-Western India. New Delhi: Oxford University Press, 217pp.

Furness, R.W. \& J. Greenwood (1993). Birds as Monitors of Environmental Change. Chapman and Hall, London, 356pp.
Grimmett, R., C. Inskipp \& T. Inskipp, (2013). Birds of the Indian Subcontinent. Oxford University Press, New Delhi, 528pp.

Islam, M.Z., \& A.R. Rahmani, (2004). Important Bird Areas in India: priority sites for conservation. Indian Bird Conservation Network, Bombay Natural History Society and Birdlife International (UK), 1-1200pp.

IUCN (2015). BirdLife International. Ciconia ciconia. 2015, IUCN Red List of Threatened Species. Downloaded on 06 May 2016.

Kazmierczak, K. (2000). A Field Guide to the Birds of the Indian Subcontinent. A \& C Black Publishers Ltd., Pica press, 352pp.

Kumar, A. (2003). Acoustic communication in birds. Resonance 8(6): 44-55.

Kumar, A., J.P. Sati, P.C. Tak \& J.R.B. Alfred (2005). Handbook on Indian Wetland Birds and their Conservation. Zoological Survey of India, 218pp.

Li, L., D. Hu, D. Duan, E.Gong \& L. Hou (2009). Alethoalaornithidae: a new family of enantiornithine bird from the Lower Cretaceous of Western Liaoning. Acta Palaeontologica Sinica 46(3): 365-372

Manakadan, R. \& A. Pittie (2001). Standardised common and scientific names of the birds of the Indian subcontinent. Buceros 6(1): 1-37.

Nagelkerken, I., S.J.M. Blaber, S. Bouillon, P. Green, M. Haywood, L.G. Kirton, J.O. Meynecke, J. Pawlik, H.M. Penrose, A. Sasekumar \& P.J. Somer-field (2008). The habitat function of mangroves for terrestrial and marine fauna: A review. Aquatic Botany 89: 155-185; http://dx.doi.org/10.1016/j.aquabot.2007.12.007

Newton, I., (1995). The contribution of some recent research on Birds to ecological understanding. Journal of Animal Ecology 64: 675-696.

Nitsure, S.R. \& M. Pejaver (2002). Species diversity of avifauna at Thane Creek near Rutuchakra Nature Park. Proceeding of National Seminar on Creeks, Estuaries and Mangroves-Pollution and Conservation, 276-282pp.

Oswin, S.D. (2002). Biodiversity and ecology of the Gulf of kutch Mangroves, Gujarat. Proceeding of National Seminar on Creeks, Estuaries and Mangroves-Pollution and Conservation, 78-83pp.

Pawar, P.R. (2011). Species diversity of birds in mangroves of Uran (Raigad), Navi Mumbai, Maharashtra, West coast of India. Indian Journal of Experimental Sciences 2(10): 73-77.

Rotenberry J.T. \& J.A.Wiens (2009). Habitat relations of shrubsteppe birds: a 20-year retrospective habitat. The Condor 111: 401-413; http://dx.doi.org/10.1525/cond.2009.090015

Vijayan, V.S. (1991). Keoladeo National Park Ecology Study: Summary Report 1980-1990. Bombay Natural History Society, Mumbai, 57pp.

Wiens, J.A. (1995). Habitat fragmentation: island vs. landscape perspectives on bird conservation. Ibis. 137pp; http://dx.doi. org/10.1111/j.1474-919X.1995.tb08464.x 